\title{
High spatial resolution performance of a triple Fabry-Pérot filtergraph
}

\author{
O. von der Lühe and Th.J. Kentischer \\ Kiepenheuer-Institut für Sonnenphysik, Schöneckstraße 6-7, D-79104 Freiburg i. Br., Germany \\ e-mail: ovdluhe@kis.uni-freiburg.de
}

Received June 8; accepted August 14, 2000

\begin{abstract}
We investigate the consequences of monochromatic pupil apodisation in a filtergraph based on FabryPérot etalons in a telecentric mount, an effect which was discovered by Beckers (1998a). The pupil apodisation gives rise to systematic changes of the point spread function in the presence of strong spectral gradients, like in the wings of a Fraunhofer line. These changes are likely to produce spurious velocity signals in Dopplergrams generated from high spatial resolution filtergrams of the solar surface. We include in our analysis for the first time the effects of optical phase changes on pupil apodisation which has the tendency to enhance velocity errors.

We study the resolution and the velocity errors for the three-etalon filtergraph TESOS (Kentischer et al. 1998) at the Vacuum Tower Telescope on Tenerife. Peak-to-valley velocity errors for solar structure with sizes of 2 arcsec and less and a contrast of $15 \%$ amount to less than $50 \mathrm{~m} / \mathrm{s}$ for the low resolution mode and to less than $10 \mathrm{~m} / \mathrm{s}$ for the high resolution mode of TESOS in the worst case. We conclude that the performance of TESOS is not severely compromised by pupil apodisation.
\end{abstract}

Key words: instrumentation: interferometers instrumentation: spectrographs

\section{Introduction}

Narrow-band tunable filtergraphs play an important role in solar physics, because only the simultaneous observation of both spatial dimensions permit studying the dynamics of small scale structure with sufficient temporal resolution. Efficient tunable filtergraphs can be constructed with Fabry-Pérot interferometers (FPIs) in combination with prefilters which select a suitable passband

Send offprint requests to: O. von der Lühe within the channeled spectrum. Several filtergraphs using FPIs have been recently constructed for solar observations (Bonaccini et al. 1989; Bendlin et al. 1992; Cavallini 1998; Kentischer et al. 1998). The spectral passband of an FPI varies with the angle of incidence of a plane wave which transverses the interferometer. In all designs except the one by Bendlin et al. (1992), the FPIs are operated in a telecentric configuration - i.e., with a collimated pupil - in order to maintain the passband in the same position at all field points. The consequence is a slight reduction in spectral resolution because the central wavelength of the passband varies across the pupil. This reduction is accounted for in the specification of the FPI to meet the design goal of the filtergraph.

Beckers (1998a, 1998b) has investigated the effects of the passband variation across the pupil on high spatial resolution spectrometry. In monochromatic light, the variation causes an apodisation within the pupil which modifies the point spread function (PSF) in the image plane compared to the PSF of a uniformly illuminated pupil. We refer to this modification as pupil apodisation in the following. As Beckers points out, spectral variations of the PSF in combination with the intensity variations of a Fraunhofer line may lead to spurious signals in Dopplergrams and may render a telecentric design unsuitable for high resolution work. Beckers limited his investigations to the variation of the magnitude of the electromagnetic field within the pupil, the corresponding effects on the field phase were not treated. The effects could therefore be even worse than descibed by Beckers.

The Telecentric Etalon SOlar Spectrometer (TESOS) is a Fabry-Pérot etalon based filtergraph which operates at the Vacuum Tower Telescope of the Observatorio del Teide on Tenerife since a few years (Kentischer et al. 1998). TESOS originally used two FPIs in a telecentric configuration; it was recently upgraded with a third FPI which is also in the telecentric beam (Kentischer et al. 2000). Since high spatial resolution - although not at the diffraction limit - is a prime science goal of TESOS, we were 


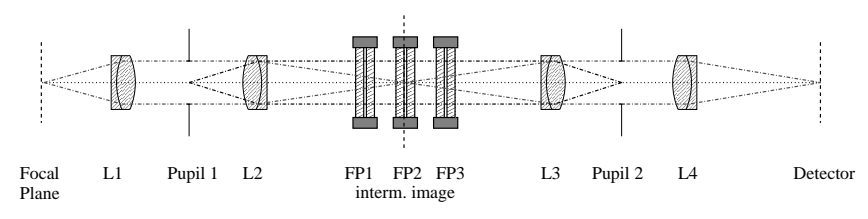

Fig. 1. Sketch of the optical layout of TESOS. L1 to L4: achromatic lenses. The telescope focal plane is reimaged by a system of a collimator and a camera lens (L1 and L2). The focal length of L2 equals its distance from Pupil 1, thus providing the telecentric beams in the intermediate image plane. A second system of collimator and camera lenses (L3 and L4) produces an image of the focal plane on the detector. FP1, FP2 and FP3 show the position of the three Fabry-Pérot etalons. The position of a prefilter wheel is in the same image space as the etalons is not indicated. The two magnifications with $F=1 / 128$ and $F=1 / 256$ are realized by an exchange of L2 and a mechanical rearrangement of the etalons

concerned by Beckers' findings. In this paper, we repeat his analyses specifically for TESOS, extend the treatment to the full complex field, and investigate the combined effects of three FPIs.

\section{Pupil apodisation of TESOS}

Figure 1 presents a sketch of the principle of the TESOS filtergraph. TESOS uses two magnifications, a low resolution mode with $0.25 \mathrm{arcsec} / \mathrm{pixel}$ and an $F$ number of $F=1 / 128$ of the beam at the position of the etalons, and a high resolution mode with 0.11 arcsec/pixel and $F=1 / 256$. The low resolution mode is far from resolving the diffraction limit of 0.15 arcsec at $\lambda=500 \mathrm{~nm}$, while the high resolution mode remains within a factor of 1.4 from critically sampling the diffraction-limited point spread function of the VTT. The pupil apodisation effects are roughly four times less in the high resolution mode compared to the low resolution mode due to the smaller numerical aperture.

The transmission $A$ for the complex electromagnetic field of a single FPI is given with the Airy function (see e.g., Tolansky 1948),

$A=T^{2} \frac{1-R^{2} \exp -i \Psi}{1+R^{4}-2 R^{2} \cos \Psi}$,

where $R$ is the amplitude reflectivity of the cavity surfaces, $T=1-R$ is the amplitude transmission of the surfaces, $\Psi$ is the optical phase delay of the cavity and $i=\sqrt{-1}$.

The phase delay for a wave with wavelength $\lambda$ and angle of incidence $\alpha$ is given with

$\Psi=2 \pi \frac{2 n(d+\delta)}{\lambda} \cos \alpha$,

where $n$ is the index of refraction of the medium inside the cavity, $d$ is the nominal width of the cavity, and $\delta$ is a tuning parameter which is adjusted such as to make the
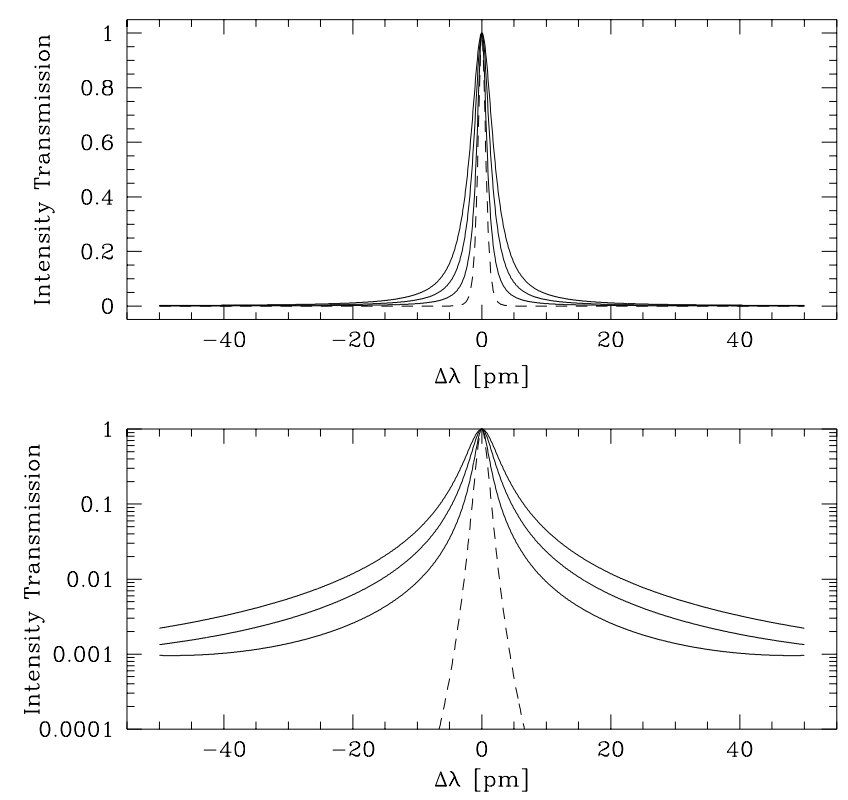

Fig. 2. Spectral passband of TESOS for $\alpha=0^{\circ}$ in linear (top) and logarithmic scales (bottom). Solid lines represent the transmission of the individual etalons, the dashed curve represents the combination of the three FPIs

phase delay an integer multiple of $2 \pi$ for the desired center wavelength $\lambda_{\mathrm{c}}$ of the passband at $\alpha=0$.

The cavity width of the widest etalon in TESOS is $d_{1}=1.3 \mathrm{~mm}$, the others are narrower by factors of $\epsilon_{2}=0.617$ and $\epsilon_{3}=0.439$. The tuning parameters $\delta_{1}$, $\delta_{2}$ and $\delta_{3}$ are computer controlled to make the maxima of the spectral channels coincide at any desired wavelength within a range from $430 \mathrm{~nm}$ to $750 \mathrm{~nm}$. The superposition results in a $2.5 \mathrm{pm}$ wide overall passband, the spectral channels remain incommensurable for a spectral range of about $3 \mathrm{~nm}$. Interference filters with $1 \mathrm{~nm}$ passbands select the desired superposition of channels within the region of interest in the spectrum. The passbands of the three etalons are scanned successively across a spectral line with typical step sizes of $2.5 \mathrm{pm}$ (equivalent to $1.5 \mathrm{~km} \mathrm{~s}^{-1}$ ). A filtergram is taken at each wavelength position simultaneously with an image of the observed region in the continuum; the latter serves for precise registration of the filtergrams during data analysis.

Figure 2 shows the individual passbands of the FPIs as well as their superposition for a central wavelength $\lambda_{\mathrm{c}}=500 \mathrm{~nm}$ and for $\alpha=0^{\circ}$ according to Eq. (1). The medium inside the cavities is air for which we assume $n=1$. The separation of the FPIs is $20 \ldots 30 \mathrm{~cm}$, so we disregard any interference effects between etalons.

We compute the combined complex field transmission by multiplication of the individual transmissions of the etalons. If we take the same reflectivity $R$ for all surfaces, 
we obtain

$$
\begin{aligned}
A_{\text {tot }}=T^{6} & \left(\frac{1-R^{2} \exp \left[-i \Psi_{1}\right]}{1+R^{4}-2 R^{2} \cos \Psi_{1}}\right) . \\
& \left(\frac{1-R^{2} \exp \left[-i \Psi_{2}\right]}{1+R^{4}-2 R^{2} \cos \Psi_{2}}\right) . \\
& \left(\frac{1-R^{2} \exp \left[-i \Psi_{3}\right]}{1+R^{4}-2 R^{2} \cos \Psi_{3}}\right),
\end{aligned}
$$

where the phase delays $\Psi_{1}, \Psi_{2}, \Psi_{3}$ apply to the three FPIs, i.e.,

$$
\begin{aligned}
& \Psi_{1}=2 \pi \frac{2 n\left(d_{1}+\delta_{1}\right)}{\lambda} \cos \alpha, \\
& \Psi_{2}=2 \pi \frac{2 n\left(\epsilon_{2} d_{1}+\delta_{2}\right)}{\lambda} \cos \alpha, \\
& \Psi_{3}=2 \pi \frac{2 n\left(\epsilon_{3} d_{1}+\delta_{3}\right)}{\lambda} \cos \alpha .
\end{aligned}
$$

The transmission computed in this manner applies to a single ray, representing a plane wavefront at wavelength $\lambda$, incident on the etalons under the same angle $\alpha$. Inspection of Fig. 1 reveals that a plane wave transmitted by the etalons corresponds to light which emerges from a point in Pupil 1. Since the phase delay (Eq. 2) varies with the cosine of the angle of incidence, the overall transmission peak wavelength shifts slightly across the pupil. The result is a variation in transmission across the pupil for monochromatic light which depends on the numerical aperture $F$ of the beams at the intermediate image plane. This effect also broadens the transmission profile of the filtergraph and shifts the central wavelength slightly towards the blue by a factor of $\cos F / 3$.

Figure 3 shows in the left panels the logarithm of the monochromatic intensity and the phase across the pupil diameter as seen through the FPIs, and as a function of wavelength for $\pm 12.5 \mathrm{pm}$ about $\lambda_{\mathrm{c}}=500 \mathrm{~nm}$ and for $F=1 / 128$ (the effects for $F=1 / 256$ are similar, but considerably smaller). The right panels show the variation of intensity and phase at a fixed position in wavelength corresponding to the shifted peak of the transmission profile, across the two-dimensional pupil. When the wavelength is scanned from the blue to the red side of the passband, the intensity first increases near the pupil edges as a ring of light which shrinks as the wavelength increases until the light patch disappears at the pupil center redwards of the peak transmission.

\section{High resolution performance of TESOS}

Any variation of the complex field across the pupil modifies the point spread function (PSF) at the position of the detector, which is readily computed from Fourier optics principles. Let $A_{\text {tot }}(\alpha)$ denote the - circularly symmetric - complex transmission of the filtergraph as a function of angle of incidence, then the radial distribution $p_{\lambda}(\rho)$ of the complex field in the image plane at wavelength $\lambda$ is
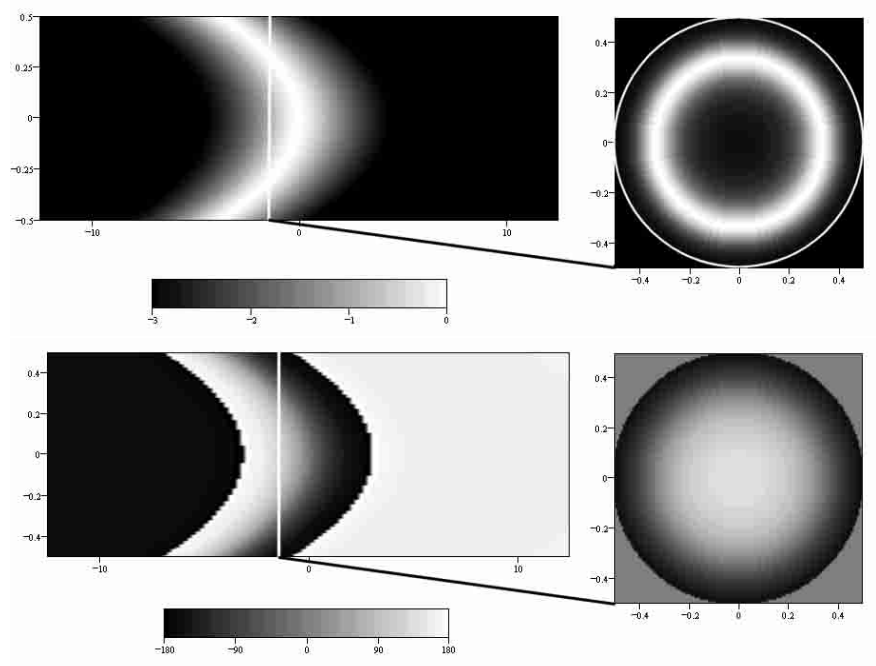

Fig. 3. Top left: three decades of intensity in a log scale as a function of wavelength across the pupil diameter for $F=$ $1 / 128$. The horizontal scale is the wavelength departure from $\lambda_{\mathrm{c}}=500 \mathrm{~nm}$ in $\mathrm{pm}$. The vertical scale represents pupil position in units of pupil diameter. Top right: intensity distribution inside the pupil in a linear scale at the wavelength indicated by the vertical line in the left panel. The white circle on the right shows the edge of the pupil. Bottom: wrapped pupil phase as a function of wavelength across the pupil diameter (left) and within the pupil (right). The scale represents values between $\pm 180^{\circ}$

given with

$$
\begin{aligned}
p_{\lambda}(\rho) & =\mathcal{H}\left[A_{\text {tot }}(\alpha)\right] \\
& =2 \pi \int_{0}^{F / 2} A_{\text {tot }}(\alpha) J_{0}\left(2 \pi \frac{\alpha \rho}{\lambda}\right) \mathrm{d} \alpha .
\end{aligned}
$$

$\mathcal{H}[\ldots]$ denotes the Hankel transform (Bracewell 1986) of its argument and $J_{0}$ is the zeroth-order Bessel function of the first kind. For light at wavelength $\lambda$, the monochromatic PSF is given with $I_{\lambda}(\rho)=\left|p_{\lambda}(\rho)\right|^{2}$.

Figure 4 shows the monochromatic PSF for a range of wavelengths across the transmission band of TESOS. The PSF is quite sharp but shows broad wings of fringes bluewards of the transmission peak. They occur when the monochromatic transmission within the pupil concentrates near the edge, representing an annulus of light, and in combination with the fluctuations of the optical phase. The latter effect was not taken into account by Beckers. The illuminated part of the pupil is concentrated near its center redwards of $\lambda_{c}$, reducing the effective telescope aperture and causing a broadening of the core of the PSF. This figure compares qualitatively with Fig. 1 of Beckers (1998a), except for the enhancement in the blue wings.

In order to be able to compare the width of the monochromatic PSF $p_{\lambda}(\rho)$ with the width of the PSF $p_{0, \lambda}(\rho)$ for a uniformly illuminated pupil, we define the "equivalent radius" $h_{\lambda}$ at wavelength $\lambda$ as

$h_{\lambda}=\frac{\int_{0}^{\infty} p_{\lambda}(\rho) \mathrm{d} \rho}{p_{\lambda}(0)}$, 

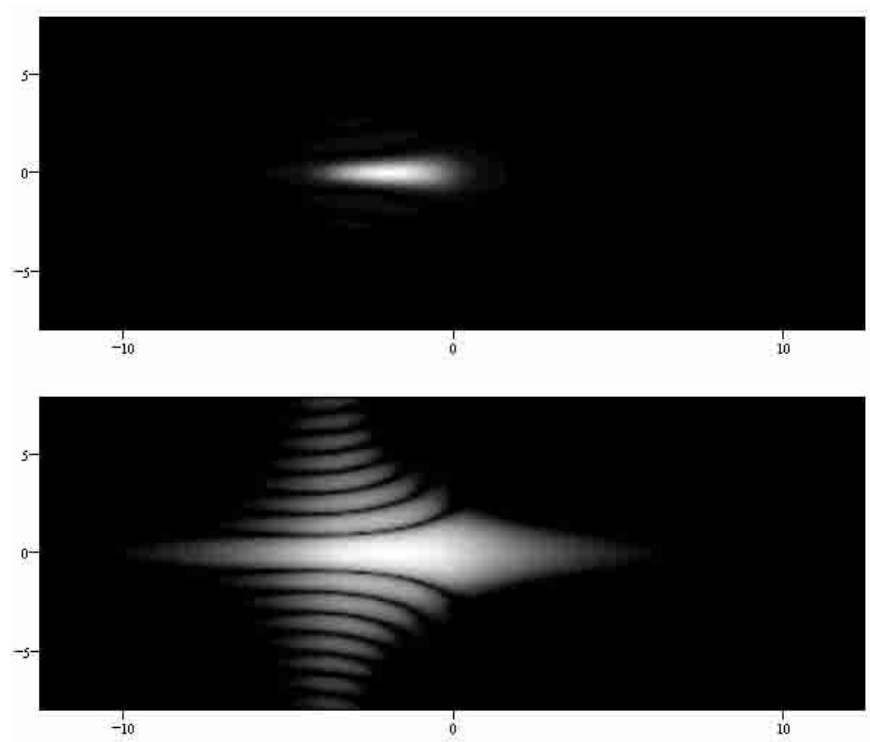

Fig. 4. Top: monochromatic point spread function as a function of wavelength for the low resolution $F=1 / 128$ mode of TESOS (linear scale). Bottom: same as above, but for four decades of intensity in a log scale. The brightness scale represents values between 0 and 1 . The horizontal scale is wavelength departure from $\lambda_{\mathrm{c}}=500 \mathrm{~nm}$ in $\mathrm{pm}$. The vertical scale represents image position in units of $\lambda_{c} / D$

noting that the PSF has a maximum for $\rho=0$. We then compute the relative equivalent radius $\epsilon_{\lambda}$ from the equivalent radius $h_{0, \lambda}$ for the case of a uniformly illuminated pupil as follows,

$\epsilon_{\lambda}=\frac{h_{\lambda}-h_{0, \lambda}}{h_{\lambda}+h_{0, \lambda}}$

Figure 5 shows $\epsilon_{\lambda}$ as a function of wavelength for both magnifications. It is seen that for the low magnification of TESOS, there is a variation of the PSF width of up to $50 \%$ compared with the uniformly illuminated pupil PSF, while for the high magnification the effect amounts to just a few \%. It is this variation which causes the spurious velocity signals when filtergrams in different wings are compared.

The polychromatic point spread function is calculated by taking the squared modulus of the complex field distribution at each wavelength and then integrating over the transmission band of the filtergraph (cf. Fig. 2). The detected intensity distribution $I(\rho)$ of a point source in the absence of any additional aberrations in the system is given as

$I(\rho)=\int_{\lambda_{\mathrm{c}}-\Delta \lambda / 2}^{\lambda_{\mathrm{c}}+\Delta \lambda / 2} S(\lambda)\left|p_{\lambda}(\rho)\right|^{2} \mathrm{~d} \lambda$,

where $S(\lambda)$ is the intensity of the point source at wavelength $\lambda, \lambda_{\mathrm{c}}$ is the wavelength of maximum transmission of the filtergraph and $\Delta \lambda$ is chosen such as to fully include the filtergraph passband. If the spectrum of the source
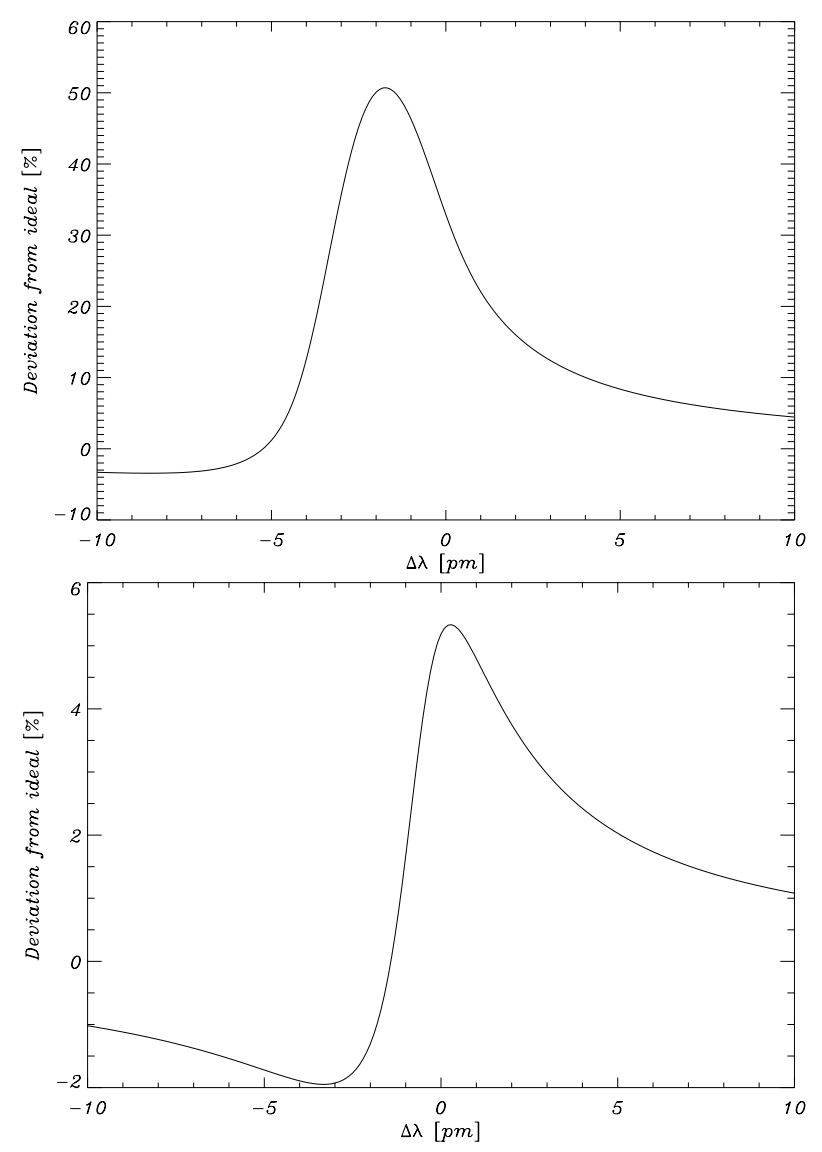

Fig. 5. Relative equivalent radius of the point spread function the low resolution mode (top) and high resolution mode (bottom) at a wavelength of $500 \mathrm{~nm}$

is a continuum, $S$ is a constant and can be taken outside the integral in Eq. (8) and the PSF will remain constant for small changes of $\lambda_{c}$. The corresponding polychromatic PSF is shown in Fig. 6 along with the point spread function from a uniformly illuminated pupil. The filtergraph PSF differs from the uniformly illuminated pupil PSF mainly by the strong, fringed halo which is caused by the annular illumination of the pupil in the blue wing. The effect is particularly strong for the low resolution mode, where most of the intensity is actually in the wings of the PSF. The Strehl'sche Definitionshelligkeit (ratio of peak intensities of aberrated and unabberated PSFs) is as low as 0.16 in this case. The halo for the high resolution mode is much smaller and the Strehl ratio of the PSF is 0.81 . The full width at half maximum (FWHM) of the $\mathrm{PSF}$ is independent of the magnification.

Figure 7 shows the modulation transfer functions of TESOS which result from the polychromatic point spread functions of both magnifications. It is evident that the resolution of the instrument is seriously affected when the numerical aperture is large, as is the case for the low magnification. Note the high frequency tail which extends to the diffraction limit at a level of about 0.05 . The high resolution mode with the less divergent beams suffers much 

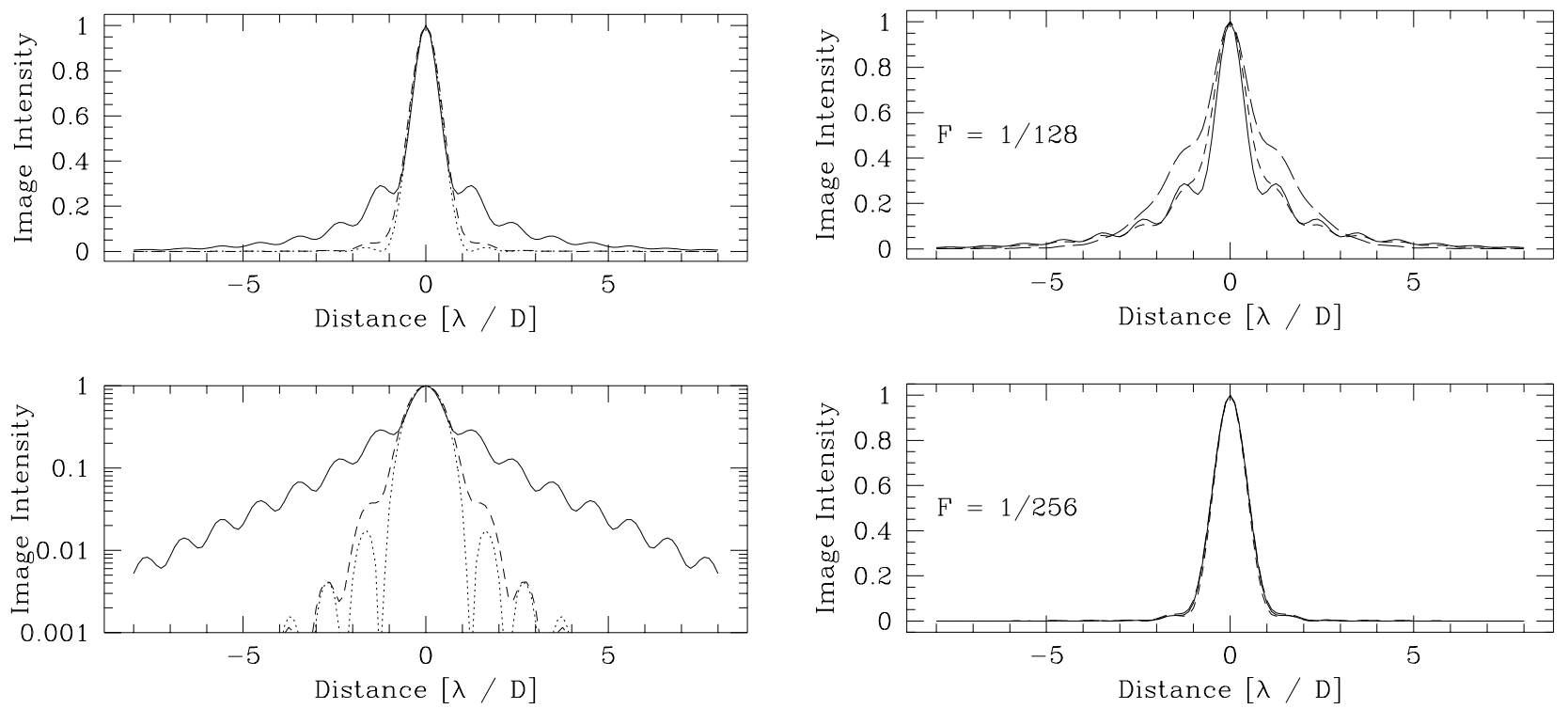

Fig. 6. Top left: polychromatic PSF for spectrally flat sources at $\lambda_{\mathrm{c}}=500 \mathrm{~nm}$. The PSF for low (solid) and high resolution modes (dashed), and the PSF for a uniformly illuminated pupil (dotted) are shown in a linear scale. Bottom left: same as above, but for three decades of intensity in a log scale. Top right: polychromatic PSF for the spectral line with the filtergraph tuned to line center (solid curve), to the blue wing (short dashed), and to the red wing (long dashed), for the low resolution mode $(F=1 / 128)$. Bottom right: same as above, but for the high resolution mode $(F=1 / 256)$. The horizontal scale is image position in units of $\lambda_{\mathrm{c}} / D$

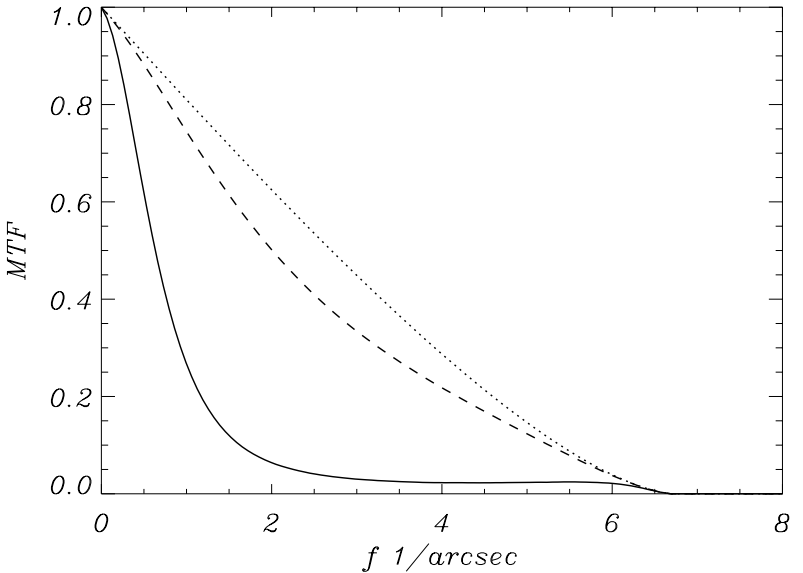

Fig. 7. Polychromatic modulation transfer function (MTF) for the low resolution (solid line) and high resolution (dashed line) modes. The dotted line represents the MTF for absence of pupil effects

less and differs only little from the theoretical case without pupil effects.

We computed the polychromatic point spread function without the phase variation in the pupil to be able to compare our treatment with Beckers' original results. Figure 8 presents the polychromatic PSF with and without the pupil phase variation for the low resolution mode. It is evident that ignoring the phase leads to much less pronounced wings and significantly underestimates the apodisations effect.

\section{Errors in velocity measurements}

When scanning a spectral line, the intensity varies across the passband of the filtergraph for any given setting of $\lambda_{\mathrm{c}}$. Depending on the position of the center wavelength of the filtergraph, either the blue or the red side of the transmission profile receives more light. We have calulated the effect for a spectral absorption line with a Gaussian profile of width $\Delta \lambda_{\mathrm{L}}=6.5 \mathrm{pm}$ and core intensity $I_{\text {core }}=0.3$,

$S(\lambda)=I_{\text {core }}+\left(1-I_{\text {core }}\right)\left(1-\exp -\frac{\left(\lambda-\lambda_{0}\right)^{2}}{2 \Delta \lambda_{\mathrm{L}}^{2}}\right)$,

where the filter was tuned to three positions $\lambda_{\mathrm{c}}=\lambda_{0}$, $\lambda_{0}-\Delta \lambda_{\mathrm{L}}$, and $\lambda_{0}+\Delta \lambda_{\mathrm{L}}$. The corresponding point spread functions are shown for both magnifications in the right column of Fig. 6.

There are evident differences for the low resolution mode. The line core PSF $I_{\mathrm{C}}(\rho)$ resembles the PSF for a flat spectrum. The PSF $I_{\mathrm{B}}(\rho)$ at the blue line wing has a relatively sharp core with extended wings while the red line wing PSF $I_{\mathrm{R}}(\rho)$ is broader in the center with less intense wings. The high resolution mode PSFs are essentially the same for all positions in the spectral line.

Pupil apodisation would not do any harm as long as the observed source was featureless. Spurious spectral features could be produced when there are fluctuations of intensity, and could be misinterpreted. In particular, structure-dependent displacements of a Fraunhofer line of a medium which is at rest relative to the observer could be interpreted as Doppler shifts. We studied the magnitude of such an error by including a sinusodial intensity variation whose period and contrast was varied into the source 

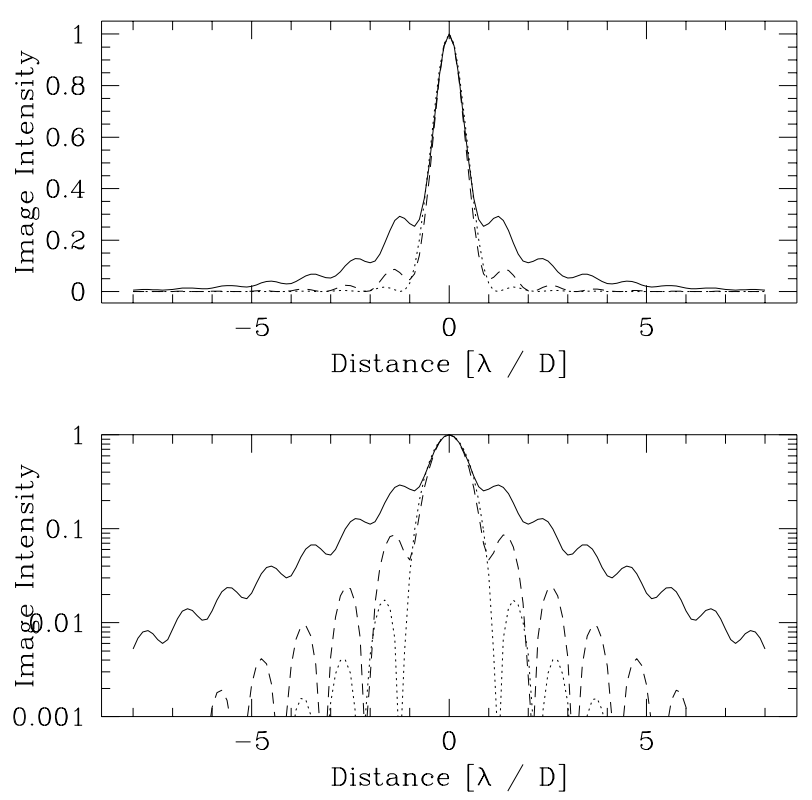

Fig. 8. Top: polychromatic PSF for spectrally flat sources at $\lambda_{\mathrm{c}}=500 \mathrm{~nm}$ for the low resolution mode with a linear scale. Solid line: PSF including pupil phase variation. Dashed line: PSF with pupil phase variation ignored. The PSF for a uniformly illuminated pupil is shown as the dotted line. Bottom: same as above, but for three decades of intensity in a log scale

model. The intrinsic velocity of the modelled structure was zero. The spectra were analysed for Doppler shifts, any deviation from zero Doppler velocity was interpreted as a velocity error. It turns out that spurious Doppler signals also show sinusoidal modulation of the same period as the intensity modulation, but with a period-dependent phase relative to the intensity modulation.

Figure 9 shows the amplitude and phase variation of the velocity error with the period of intensity modulation for an rms contrast of $15 \%$, corresponding to a high contrast structure such as penumbral filaments. The dotted lines represent the phase between intensity fluctuation and velocity error. The velocity error in the low resolution mode approaches $25 \mathrm{~m} / \mathrm{s}$ at scales between 1 and 2 arcsec. Brighter regions appear blueshifted at these scales, which corresponds to a phase shift of $180^{\circ}$ between intensity and velocity error. A secondary maximum of $18 \mathrm{~m} / \mathrm{s}$ at 0.25 arcsec shows the velocity error in phase with the intensity modulation, so that brighter regions appear now redshifted. The velocity error of the high resolution mode is less than $2 \mathrm{~m} / \mathrm{s}$ for scales above 0.7 arcsec where bright regions experience blueshifts. There is a maximum of just above $6 \mathrm{~m} / \mathrm{s}$ in the presence of a spatial modulation with a scale of $0.3 \operatorname{arcsec}$, or $250 \mathrm{~km}$ on the solar surface, where bright regions experience redshifts.

Figure 10 shows the amplitude of the velocity error as a function of sinusodial modulation period and an rms contrast between 0 and $15 \%$. Velocity errors of typical granular structures with an rms contrast of some $10 \%$ and scales between 1 and 2 arcsec are of the order of $15 \mathrm{~m} / \mathrm{s}$ in the low resolution mode while they amount to less than $1 \mathrm{~m} / \mathrm{s}$ in the high resolution mode. Scales at the diffraction limit with contrasts of $10 \%$ suffer from velocity errors of less than $5 \mathrm{~m} / \mathrm{s}$.

\section{Discussion and conclusions}

We have analyzed the effects of pupil apodisation in the TESOS filtergraph on the angular resolution and accuracy of Doppler velocity measurements of solar small scale features. We confirm Beckers' observation that a telecentric mount FPI filtergraph suffers from significant pupil apodisation; even more so when also the deformation of optical phase is included in the analysis. The latter gives rise to an enhanced halo in the point spread function in the blue wing of a line when the numerical aperture is large, in comparison to calculations where the phase effects are not taken into account.

The dependence of the phase between intensity and velocity error on structure size is easy to understand. The PSF in the blue wing of a spectral line has a sharp core and broad wings compared to the PSF in the red wing where the core is broader but wings are essentially absent. A spectral line in the presence of large scale intensity fluctuations will be blurred in the blue wing because of the extended wings of the PSF and show more contrast in the red wing. The net result is an apparent blueshift in the brighter parts of the structure and an apparent redshift in the darker parts, resulting in an anti-correlation of intensity and lineshift.

In the presence of small scale intensity fluctuations, the opposite is true, because now the sharp core of the blue wing PSF wins over the broader core in the red wing, and intensity and lineshift will be correlated. Practically, there will be a mixture of scales in the observed solar structure, and the simultaneous presence of intermediate and small scales may cause correlated superposition effects which enhance the error through the phase relation. A conservative estimate of the velocity error will therefore be the sum of the peaks at intermediate and small scales. One can expect in the worst case a peak-to-valley error of $45 \mathrm{~m} / \mathrm{s}$ for the low resolution mode and of $9 \mathrm{~m} / \mathrm{s}$ for the high resolution mode and for a contrast of $15 \%$ rms. The error will scale linearly with the intensity contrast.

With respect to the performance of TESOS, we make the following observations:

1. The pupil apodisation effects of TESOS are comparable to those of a single Fabry-Pérot etalon filtergraph with the same spectral bandwith. The addition of the other two interferometers only serve to increase the free spectral range of the filtergraph;

2. The low resolution mode just performs as such. The spatial resolution is slightly better than one arcsec and 

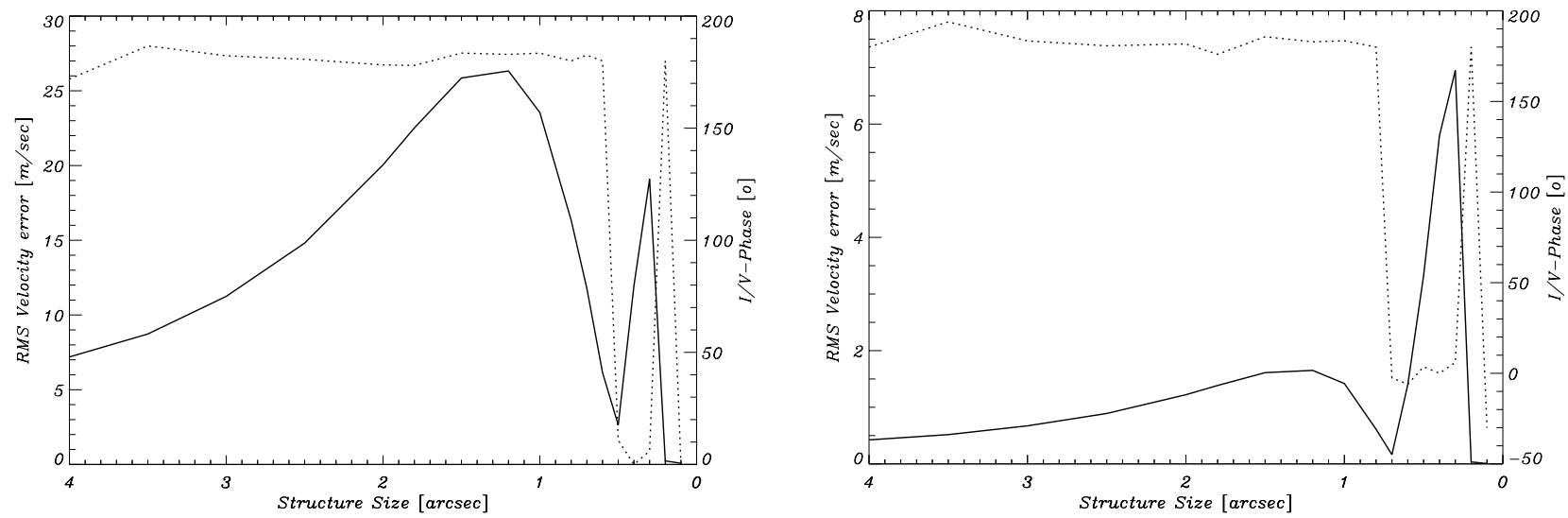

Fig. 9. Velocity error as a function of intensity modulation period for the low resolution (left) and high resolution (right) modes. The solid line represents the velocity error (scale to the left) and the dotted line the phase of the velocity error relative to the intensity modulation (scales to the right). The rms intensity contrast is $15 \%$ at all scales

is quite well adapted to the pixel scale. Although the modulation transfer function drops rapidly at scales of 1 arcsec, there is remaining signal at higher frequencies which could be recovered by deconvolution if the quality of the data warranted it;

3. The spatial resolution of the high resolution mode does not suffer significantly from pupil apodisation degradation;

4. Velocity errors in the low resolution mode are comparable to or smaller than other sources of velocity error in TESOS (see below);

5. The high resolution mode does not suffer from significant velocity errors at all scales and contrasts which are relevant to solar observations.

The random velocity error in the low resolution filtergrams of TESOS is estimated to be of the order of $100 \mathrm{~m} / \mathrm{s}$ (Schlichenmaier \& Schmidt 1999). The most significant source is the wavelength sampling of typically $2.5 \mathrm{pm}$, which corresponds to a Doppler velocity of $1.5 \mathrm{~km} \mathrm{~s}^{-1}$. Other sources are incomplete calibration of the filtergrams, straylight and detector noise. A systematic variation of seeing during a line scan gives also rise to small scale contrast-dependent spurious velocity signals when one line wing is better resolved than the other. Taken all together, these effects are worse than the errors caused by pupil apodisation.

It is conceivable that very fine structure such as small magnetic flux concentrations and umbral dots have contrasts which exceed the range that we have investigated. But even with contrasts of up to $100 \%$ the velocity error in the high resolution mode should not amount to more than $30 \mathrm{~m} / \mathrm{s}$ which is still comparable to other error sources. We therefore conclude that TESOS is very well suited for high precision and high resolution investigations of the sun.

This may not be the case for other combinations of etalon characteristics and numeric aperture in a telecetric mount filtergraph. We have therefore calculated the Strehl ratio for the TESOS filtergraph for magnifications corresponding to $\mathrm{F} / 512$ and $\mathrm{F} / 64$ to give an indication of the variation with numeric aperture. The results are shown in Fig. 11 together with the Strehl ratio of the current magnifications. It is evident that for a filtergraph with
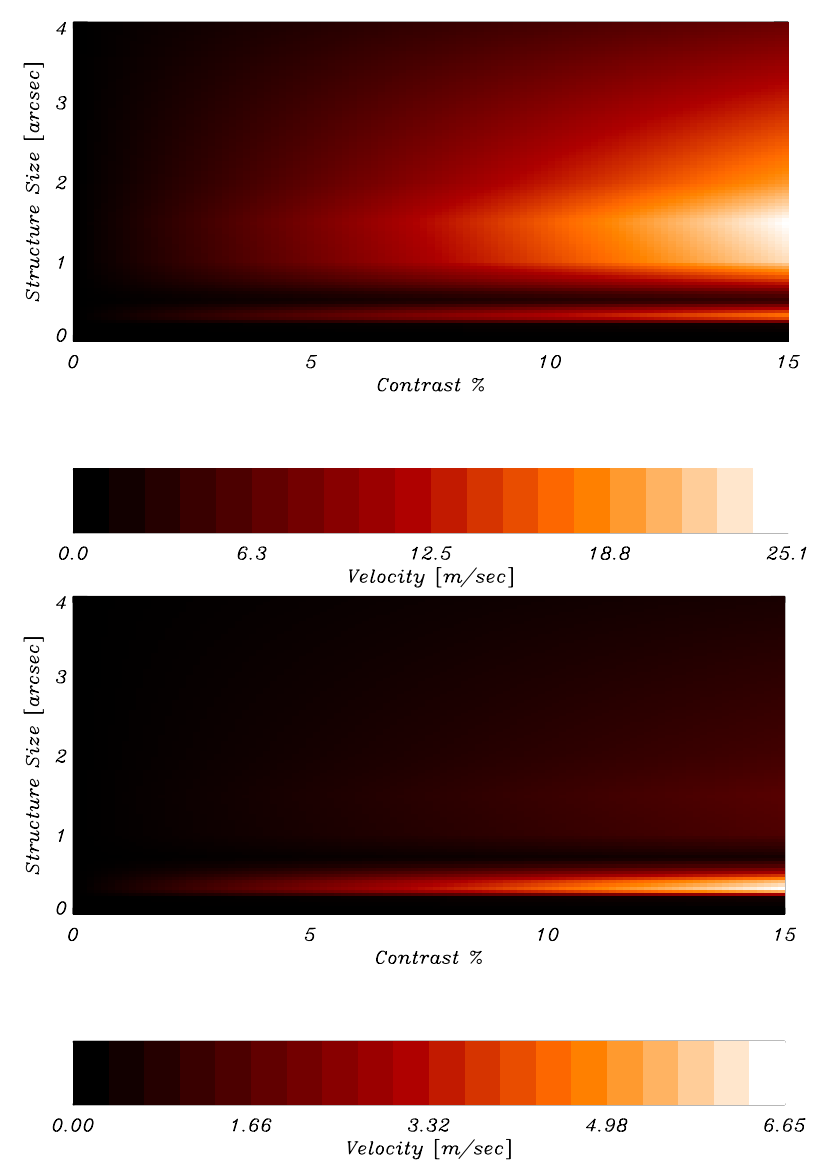

Fig. 10. Velocity error as a function of intensity modulation period for the low resolution $(F=1 / 128$, top) and high resolution $(F=1 / 256$, bottom) modes as a function of modulation period and structure contrast 


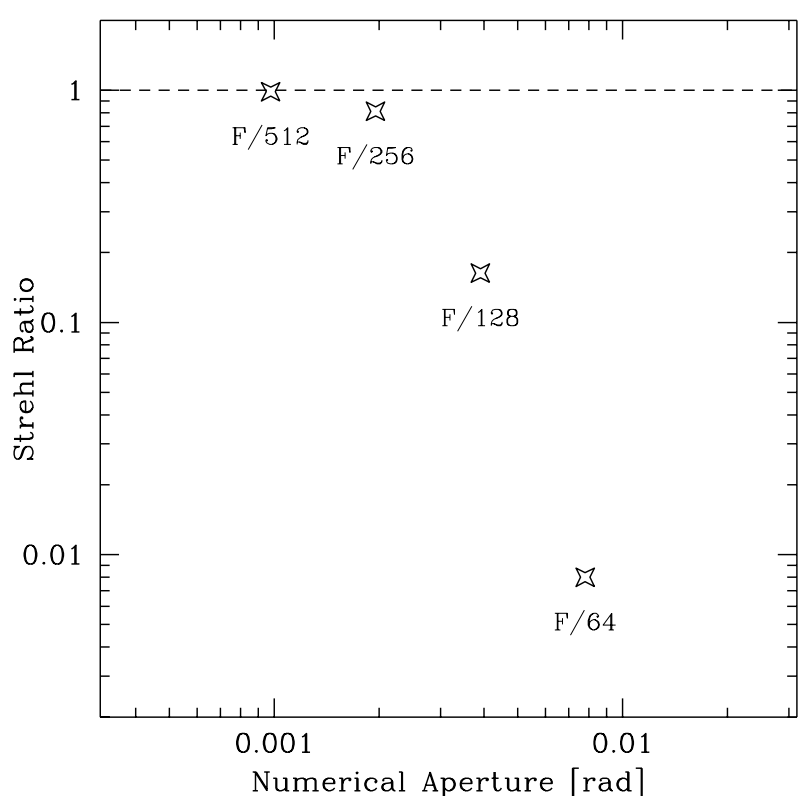

Fig. 11. Dependency of Strehl ratio on numerical aperture in the telecentric beam of TESOS

the spectral resolution of TESOS, the spatial resolution is significantly affected for numerical apertures larger than 0.002 , corresponding to $\mathrm{F} / 250$, and decays rapidly with $S \approx(N A)^{-4.4}$. Pupil apodisation effects therefore require careful consideration in the design of a filtergraph.

The alternative would be to use the etalons in a collimated configuration close to a pupil. A simple calculation shows that such a mount is not at all without problems, either. Each surface of an etalon cavity shows deviations from a perfectly flat plane at the scale of typically $\lambda / 100$ which origin from the polishing process. Microroughness at the level of a few $\mathrm{nm}$ rms adds to these deviations. Let $\Delta(\boldsymbol{r})$ be the thickness variation of the etalon's cavity as function of position $r$ perpendicular to the direction of propagation of light with $\Delta(\boldsymbol{r})<<\lambda_{\mathrm{c}}$. It is then easy to show from Eqs. (1) and (2) that the optical phase $\phi(\boldsymbol{r})$ of an originally plane wavefront transmitted by the etalon is given with

$\phi(\boldsymbol{r})=\arctan \frac{2 R^{2}}{1-R^{2}} \frac{2 \pi}{\lambda} \Delta(\boldsymbol{r})$.

For TESOS, $R^{2}=0.97$ and the resulting wavefront error would amount to about 160 times the wavefront error of a single etalon plate if all three etalons were placed in the collimated beam, or to more than a wave of aberration for optics with a quality of $\lambda / 100$. Such wavefront errors would make high spatial resolution observations impossible.

Acknowledgements. The authors wish to express their gratitude to J.M. Beckers, National Solar Observatory, for discussion and many helpful comments.

\section{References}

Beckers J.M., 1998, A\&AS 129, 191

Beckers J.M., 1998, Proc. SPIE 3355

Bendlin C., Volkmer R., Kneer F., 1992, A\&A 257, 817

Bonaccini D., Cavallini F., Ceppatelli G., Righini A., 1989, A\&A 217, 368

Bracewell R.N., 1986, The Fourier Transform and its Applications (2nd ed.). McGraw-Hill

Cavallini F., 1998, A\&AS 128, 589-598

Kentischer T., Schmidt W., Sigwarth M., v. Uexküll M., 1998, A\&A 340, 569-578

Kentischer T., Schmidt W., 2000 (in preparation)

Schlichenmaier R., Schmidt W., 1999, A\&A 349, L37-L40

Tolansky S., 1948, Multiple-beam Interferometry of Surfaces and Films, 1st ed. Oxford University Press, London 